\title{
Normal Fermi- Walker Derivative
}

\author{
Özgür Keskin* and Yusuf Yaylı
}

(Communicated by Bülent ALTUNKAYA)

\begin{abstract}
In this paper, first, we defined normal Fermi-Walker derivative and applied for adapted frame. Normal Fermi-Walker parallelism, normal non-rotating frame and Darboux vector of normal Fermi-Walker derivative by using normal Fermi-Walker derivative are given for adapted frame. Being conditions of normal Fermi-Walker derivative and normal non-rotating frame are researched throughout curve for Frenet frame and Adapted frame. It is shown that vector field which take part in [13] is normal FermiWalker parallel in accordance with the normal Fermi-Walker derivative along the general helix. Also, we show that the Frenet frame is normal non-rotating frame in accordance with the normal Fermi-Walker derivative. Afterwards, we testified that the adapted frame is normal non-rotating frame throughout the general helix.
\end{abstract}

Keywords: Frenet frame; Darboux frame; Non-rotating frame; Fermi-Walker derivative.

AMS Subject Classification (2010): 53A04, 53A05, 53Z05, 57R25P.

${ }^{*}$ Corresponding author

\section{Introduction}

Fermi-Walker transport is a process used to define a coordinate system or reference frame in general relativity. All the curvatures in the reference frame is due to the presence of mass-energy density. These curvatures are not arbitrary spin or rotation of the frame. Fermi-Walker derivative, Fermi-Walker parallelism and non-rotating frame expresses are identified according to this derivative for Bishop and Frenet frames. Then, the obtained notions are applied for Lie groups in $\mathbb{E}^{4}$ and are expressed on any hypersurface in $\mathbb{E}^{n+1}$ [9], [10], [11]. Also, unit vector fields according to Fermi-Walker transported are proved along Rytov-Legendre curves. Slant and Legendre curves are defined in three-dimensional warped products and are characterised via the scalar products between the normal of these curves and the vertical vector field [5], [4]. The constant precession curve is expressed as unit-speed curve that its central returns approximate a stable axis with stationary angle and stationary speed. The arc-length parametrised closed-shape solution of the native equations is obtained according to this constant precession curves with the help of direct geometric analysis [12]. Spherical images of a curve are investigated and is called a C-slant helix. Furthermore, some new characterizations for the C-slant helix are given and showed that the C-constant precession curve is a C-slant helix [13]. In recent years, some application is made by using the normal of slant helix. In the light of these opinions, we defined derivative according to the normal of curve.

Fermi-Walker derivative is given with the help of tangent vector of curve. We defined new normal FermiWalker derivative by using the normal vector of any curve in Frenet frame. In our study, normal Fermi-Walker derivative, normal Fermi-Walker parallelism, normal non-rotating frame and Darboux vector of normal FermiWalker derivative are given for adapted frame. Also, we show that the Frenet frame is non-rotating frame according to the normal Fermi-Walker derivative for any curve. On the contrary, $\{N, C, W\}$ frame is not non-rotating frame. The Fermi-Walker definitions can be defined by the first vectors of other frames.

$\overline{\text { Received : 18-October-2016, Accepted : 01-February-2017 }}$ 


\section{Preliminaries}

Fistly, we give some basic notions about curves. We define a new derivative.

Let $\mathrm{X}$ be a vector field in $\mathbb{R}^{3}$ and $\beta(s)$ be a unit-speed curve in space.

$$
\frac{\widetilde{\nabla} X}{\widetilde{\nabla} s}=\frac{d X}{d s}-\langle T, X\rangle M+\langle M, X\rangle T,
$$

that is to say, $\frac{\tilde{\nabla} X}{\nabla s}$ derivative is called Fermi-Walker derivative. Hereby $\mathrm{T}=\frac{d \alpha}{d s}, \mathrm{M}=\frac{d T}{d s}[2]$.

Furthermore, if

$$
\frac{\widetilde{\nabla} X}{\widetilde{\nabla} s}=0
$$

$X$ vector field is entitled parallel according to the Fermi-Walker derivative throughout the curve [2].

Let $\beta(s)$ be a unit-speed curve and a $\{P, R, Z\}$ be orthonormal vectors. If

$$
\frac{\tilde{\nabla} P}{\widetilde{\nabla} s}=0, \quad \frac{\tilde{\nabla} R}{\widetilde{\nabla} s}=0, \quad \frac{\tilde{\nabla} Z}{\widetilde{\nabla} s}=0,
$$

$\{P, R, Z\}$ is entitled non-rotating frame [1].

Let $\beta: I \subset \mathbb{R} \rightarrow \mathbb{E}^{3}$ is a unit-speed curve. Denote by

$$
\{N, C, N \wedge C=W\}
$$

the moving adapted frame throughout the curve $\beta(s)$ in $\mathbb{E}^{3}$.The adapted frame equations are

In [13],

$$
\left[\begin{array}{c}
N^{\prime}(s) \\
C^{\prime}(s) \\
W^{\prime}(s)
\end{array}\right]=\left[\begin{array}{ccc}
0 & f(s) & 0 \\
-f(s) & 0 & g(s) \\
0 & -g(s) & 0
\end{array}\right]\left[\begin{array}{c}
N(s) \\
C(s) \\
W(s)
\end{array}\right]
$$

$$
\begin{array}{r}
C=\frac{N^{\prime}}{\left\|N^{\prime}\right\|}, \\
W=\frac{\tau T+\kappa B}{\sqrt{\kappa^{2}+\tau^{2}}}, W=N \wedge C, \\
f=\sqrt{\kappa^{2}+\tau^{2}}, \\
g=\sigma f .
\end{array}
$$

Here, $\kappa$ is the curvature and $\tau$ is the torsion of the curve $\beta$ in the way of Frenet's frame. $f$ and $g$ are curvatures of the curve $\alpha$ in the way of the adapted frame.

Fermi-Walker derivative is given with the help of the tangent vector of curve. We will give the following definition by using the normal vector of curve for the first time in our study. We will say normal Fermi-Walker derivative name of this definition.

Definition 2.1. Let $X$ be a vector field in $\mathbb{R}^{3}$ and $\beta(s)$ be a unit-speed curve in space.

$$
\frac{\tilde{D} X}{\tilde{D} s}=\frac{d X}{d s}-\langle N, X\rangle N^{\prime}+\left\langle N^{\prime}, X\right\rangle N,
$$

defined as $\frac{\tilde{D} X}{\tilde{D} s}$ derivative is entitled normal Fermi-Walker derivative. Hereby, $\mathrm{N}=\frac{T^{\prime}}{\left\|T^{\prime}\right\|}$ is a normal vector of Frenet frame.

If

$$
\frac{\tilde{D} X}{\tilde{D} s}=0
$$

$\mathrm{X}$ vector field is called normal Fermi-Walker parallel in accordance with the normal Fermi-Walker derivative throughout the curve. 


\section{Adapted Frame and Normal Fermi-Walker Derivative}

In this section, firstly we give a different definition of the normal Fermi-Walker derivative with the help of the adapted frame. Then, we give some theorems and results.

Lemma 3.1. Let $X$ be a vector field throughout the $\beta(s)$ space curve, normal Fermi-Walker derivative can be defined of the

$$
\frac{\tilde{D} X}{\tilde{D} s}=\frac{d X}{d s}+f(X \wedge W)
$$

Proof.

$$
\frac{\tilde{D} X}{\tilde{D} s}=\frac{d X}{d s}-\langle N, X\rangle \frac{d N}{d s}+\left\langle\frac{d N}{d s}, X\right\rangle N,
$$

If $\frac{d N}{d s}=f C$ substitutes and the necessary actions are done,

$$
\frac{\tilde{D} X}{\tilde{D} s}=\frac{d X}{d s}+f(X \wedge W) .
$$

is obtained.

We now take into account a space curve which changes in time, so $X=X(s, u)$, where $\mathrm{s}$ is arc parameter and $\mathrm{u}$ is time parameter. In addition to the adapted frame equations, where $\mathrm{f}$ and $\mathrm{g}$ are dependent on both $\mathrm{s}$ and $\mathrm{u}$. We can be written the related equations composing the kinematics of the time change of the triad $\{N, C, W\}$, just as the adapted frame equations[1]:

$$
N_{u}=r C+h W, \quad C_{u}=-r N+g_{0} W, \quad W_{u}(s)=-h N-g_{0} C .
$$

The scalars $\mathrm{r}, \mathrm{h}, g_{0}$ are functions of both $\mathrm{s}$ and $\mathrm{u}$.

Once more, we may express the conception of normal Fermi-Walker derivative along the "temporal curve" parametrized by $u$. From equation (3.2), we attain the following similarities of equations in Corollary(3.4) and (3.1):

$$
\frac{\tilde{D} X}{\tilde{D} u}=\frac{d X}{d u}-r(W \wedge X)+h(C \wedge X),
$$

and

$$
\frac{\tilde{D} N}{\tilde{D} u}=0, \quad \frac{\tilde{D} C}{\tilde{D} u}=g_{0} W, \quad \frac{\tilde{D} N}{\tilde{D} u}=-g_{0} C .
$$

Thus, since one moves from $\mathrm{u}$ to $\mathrm{u}+\mathrm{du}$ for a fixed $\mathrm{s}$, the $(\mathrm{C}, \mathrm{W})$ plane evolves by an angle $g_{0} d u$.

Now we consider the conditions that a vector field is normal Fermi-Walker parallel according to the normal Fermi-Walker derivative.

Theorem 3.1. Let $\beta(s)$ be unit-speed a curve. Afterwards $X=\lambda_{1} N+\lambda_{2} C+\lambda_{3} W$ vector field is normal Fermi-Walker parallel according to the normal Fermi-Walker derivative throughout this curve iff:

$$
\begin{array}{r}
\lambda_{1}(s)=\text { constant }, \\
\lambda_{2}(s)=c_{1} \cos \left(\int_{1}^{s} g(s) d s\right)+c_{2} \sin \left(\int_{1}^{s} g(s) d s\right), \\
\lambda_{3}(s)=c_{2} \cos \left(\int_{1}^{s} g(s) d s\right)-c_{1} \sin \left(\int_{1}^{s} g(s) d s\right) .
\end{array}
$$

Here $\lambda_{1}, \lambda_{2}, \lambda_{3}$ real parameters are continuous differentiable functions. 
Proof. $\Rightarrow: \mathrm{X}$ is normal Fermi-Walker parallel in accordance with the normal Fermi-Walker derivative throughout the curve.

$$
\begin{array}{r}
\frac{\tilde{D} X}{\tilde{D} s}=\frac{d X}{d s}+f(X \wedge W), \\
\frac{\tilde{D} X}{\tilde{D} s}=\left(\frac{d \lambda_{1}}{d s}\right) N+\left(\frac{d \lambda_{2}}{d s}-g(s) \lambda_{3}\right) C+\left(\frac{d \lambda_{3}}{d s}+g(s) \lambda_{2}\right) W .
\end{array}
$$

Since $\mathrm{X}$, vector field throughout the curve $\beta(s)$, is normal Fermi-Walker parallel in accordance with the normal Fermi-Walker derivative, $\frac{\tilde{D} X}{\tilde{D} s}=0$. So,

$$
\begin{aligned}
\frac{d \lambda_{1}}{d s} & =0 \\
\frac{d \lambda_{2}}{d s}-g(s) \lambda_{3} & =0 \\
\frac{d \lambda_{3}}{d s}+g(s) \lambda_{2} & =0 .
\end{aligned}
$$

If the equation system solve,

$$
\begin{array}{r}
\lambda_{1}(s)=\text { constant } \\
\lambda_{2}(s)=c_{1} \cos \left(\int_{1}^{s} g(s) d s\right)+c_{2} \sin \left(\int_{1}^{s} g(s) d s\right) \\
\lambda_{3}(s)=c_{2} \cos \left(\int_{1}^{s} g(s) d s\right)-c_{1} \sin \left(\int_{1}^{s} g(s) d s\right) .
\end{array}
$$

is obtained.

$$
\begin{array}{r}
\Longleftarrow X=\lambda_{1} N+\lambda_{2} C+\lambda_{3} W \text { vector field and } \theta=\left(\int_{1}^{s} g(s) d s\right), \\
\lambda_{1}(s)=\text { constant }, \\
\lambda_{2}(s)=c_{1} \cos \theta+c_{2} \sin \theta, \\
\lambda_{3}(s)=c_{2} \cos \theta-c_{1} \sin \theta .
\end{array}
$$

and

$$
\begin{array}{r}
\frac{\tilde{D} X}{\tilde{D} s}=\frac{d X}{d s}+f(X \wedge W), \\
\frac{\tilde{D} X}{\tilde{D} s}=\left(\frac{d \lambda_{1}}{d s}\right) N+\left(\frac{d \lambda_{2}}{d s}-g(s) \lambda_{3}\right) C+\left(\frac{d \lambda_{3}}{d s}+g(s) \lambda_{2}\right) W \\
\frac{\tilde{D} X}{\tilde{D} s}=0,
\end{array}
$$

is obtained. $\lambda_{i}$ parameters are constant.

Theorem 3.2. Let $\beta(s)$ be general helix. Then $X=\lambda_{1} N+\lambda_{2} C+\lambda_{3} W$ vector field is normal Fermi-Walker parallel according to the normal Fermi-Walker derivative throughout the curve. $\lambda_{1}, \lambda_{2}, \lambda_{3}$ are constant.

Proof.

$$
\begin{array}{r}
\frac{\tilde{D} X}{\tilde{D} s}=\frac{d X}{d s}+f(X \wedge W), \\
\frac{\tilde{D} X}{\tilde{D} s}=\lambda_{1}^{\prime} N+\left(\lambda_{2}^{\prime}-g \lambda_{3}\right) C+\left(g \lambda_{2}+\lambda_{3}^{\prime}\right) W \\
=g\left(\lambda_{2} W-\lambda_{3} C\right) .
\end{array}
$$


is obtained. Since $\beta(s)$ is general helix, then $g=0$. So, $\frac{\tilde{D} X}{\tilde{D} s}=0$ is obtained.

Corollary 3.1. $\{N, C, W\}$ vectors are normal Fermi-Walker parallel in accordance with the normal Fermi-Walker derivative along the general helix.

Proof. Due to Theorem 3.2,

$$
\begin{aligned}
& (a) \lambda_{1}=1, \lambda_{2}=\lambda_{3}=0 \text { for } X=N, \\
& (b) \lambda_{2}=1, \lambda_{1}=\lambda_{3}=0 \text { for } X=C, \\
& (c) \lambda_{3}=1, \lambda_{1}=\lambda_{2}=0 \text { for } X=W .
\end{aligned}
$$

Now, the relationship between the normal Fermi-Walker parallelism and Euclid parallelism is defined below.

Corollary 3.2. Let X be a vector field throughout the $\beta(s)$ space curve. Throughout the space curve of $X$, normal Fermi-Walker derivative balances derivative in accordance with the adapted frame of $X$ iff

$$
X=\lambda W
$$

$\lambda$ is constant.

Proof. Due to Lemma 3.1,

$$
\begin{aligned}
\frac{\tilde{D} X}{\tilde{D} s}=\frac{d X}{d s}+f(X \wedge W), \\
\frac{\tilde{D} X}{\tilde{D} s}=\frac{d X}{d s} .
\end{aligned}
$$

iff

$$
X=\lambda W
$$

is obtained.

$\{T, N, B\}$ frame is not non-rotating in accordance with Fermi-Walker derivative. But now, we will see that is normal Fermi-Walker non-rotating according to normal Fermi-Walker derivative of $\{T, N, B\}$ frame.

Corollary 3.3. Let $\beta(s)$ be a unit-speed curve and $\{T, N, B\}$ be the Frenet frame of $\beta(s)$. The $\{T, N, B\}$ is normal FermiWalker non-rotating frame according to normal Fermi-Walker derivative throughout the space curves.

Proof. In accordance with the normal Fermi-Walker derivative,

$$
\begin{array}{r}
\frac{\tilde{D} X}{\tilde{D} s}=\frac{d X}{d s}+f(X \wedge W), \\
\frac{\tilde{D} X}{\tilde{D} s}=\frac{d X}{d s}-\langle N, X\rangle N^{\prime}+\left\langle N^{\prime}, X\right\rangle N .
\end{array}
$$

We compute the following equations to show that is a non-rotating frame of $\{T, N, B\}$ frame according to normal Fermi-Walker derivative. From Definition 2.1, if necessary calculations are done,

$$
\begin{gathered}
\frac{\tilde{D} T}{\tilde{D} s}=\frac{d T}{d s}-\langle N, T\rangle N^{\prime}+\left\langle N^{\prime}, T\right\rangle N, \\
\frac{\tilde{D} T}{\tilde{D} s}=T^{\prime}-\langle N, T\rangle N^{\prime}+\left\langle N^{\prime}, T\right\rangle N .
\end{gathered}
$$


Since $\langle N, T\rangle=0, T^{\prime}=\kappa N$ and $N^{\prime}=-\kappa T+\tau B$,

$$
\begin{array}{r}
\frac{\tilde{D} T}{\tilde{D} s}=\kappa N-0+\langle-\kappa T+\tau B, T\rangle N, \\
\frac{\tilde{D} T}{\tilde{D} s}=0 .
\end{array}
$$

Likewise, if you do others,

$$
\begin{aligned}
& \frac{\tilde{D} N}{\tilde{D} s}=\frac{d N}{d s}-\langle N, N\rangle N^{\prime}+\left\langle N^{\prime}, N\right\rangle N=0, \\
& \frac{\tilde{D} B}{\tilde{D} s}=\frac{d B}{d s}-\langle N, B\rangle N^{\prime}+\left\langle N^{\prime}, B\right\rangle N=0 .
\end{aligned}
$$

Corollary 3.4. Let $\{N, C, W\}$ be the adapted frame of $\beta(s)$. The $\{N, C, W\}$ is normal Fermi-Walker non-rotating frame according to normal Fermi-Walker derivative iff $g=0$.

Proof. In accordance with the normal Fermi-Walker derivative,

$$
\begin{array}{r}
\frac{\tilde{D} X}{\tilde{D} s}=\frac{d X}{d s}+f(X \wedge W), \\
\frac{\tilde{D} X}{\tilde{D} s}=\frac{d X}{d s}-\langle N, X\rangle N^{\prime}+\left\langle N^{\prime}, X\right\rangle N .
\end{array}
$$

We compute the following equations to show that is a non-rotating frame of $\{N, C, W\}$ frame according to normal Fermi-Walker derivative. From Definition 2.1, if necessary calculations are done,

$$
\begin{gathered}
\frac{\tilde{D} N}{\tilde{D} s}=\frac{d N}{d s}-\langle N, N\rangle N^{\prime}+\left\langle N^{\prime}, N\right\rangle N, \\
\frac{\tilde{D} N}{\tilde{D} s}=N^{\prime}-\langle N, N\rangle N^{\prime}+\left\langle N^{\prime}, N\right\rangle N .
\end{gathered}
$$

Since $\langle N, N\rangle=1$ and $N^{\prime}=f C$,

$$
\begin{array}{r}
\frac{\tilde{D} N}{\tilde{D} s}=f C-f C+\langle f C, N\rangle N, \\
\frac{\tilde{D} N}{\tilde{D} s}=0 .
\end{array}
$$

Similarly, if you do others,

$$
\begin{gathered}
\frac{\tilde{D} C}{\tilde{D} s}=\frac{d C}{d s}-\langle N, C\rangle N^{\prime}+\left\langle N^{\prime}, C\right\rangle N=g W, \\
\frac{\tilde{D} W}{\tilde{D} s}=\frac{d W}{d s}-\langle N, B\rangle N^{\prime}+\left\langle N^{\prime}, W\right\rangle N=-g C .
\end{gathered}
$$

Here, there should be $g=0$ to be normal Fermi-Walker parallel according to normal Fermi-Walker derivative. Thus, $\beta(s)$ must be general helix. Theorem 3.2 is obtained again from the result.

We express Darboux vector of the normal Fermi-Walker derivative according to the $\{N, C, W\}$ frame. In addition, in Theorem 3.3, we examine that Darboux vector of the normal Fermi-Walker derivative is normal Fermi-Walker parallel in accordance with the normal Fermi- Walker derivative. 
Corollary 3.5. Let $\{N, C, W\}$ be the adapted frame of $\beta(s)$. Darboux vector of the normal Fermi-Walker derivative in accordance with the adapted frame is

$$
\omega_{*}=g N
$$

Proof. If the calculations are done,

$$
\begin{aligned}
& \frac{\tilde{D} N}{\tilde{D} s}=\omega_{*} \wedge N, \\
& \frac{\tilde{D} C}{\tilde{D} s}=\omega_{*} \wedge C, \\
& \frac{\tilde{D} W}{\tilde{D} s}=\omega_{*} \wedge W .
\end{aligned}
$$

As a result, if there is $\omega_{*}=g N$, it is Darboux vector of the normal Fermi-Walker derivative in accordance with the adapted frame. Now, we can define new theorem.

Theorem 3.3. Let $\beta(s)$ be a unit-speed curve and $\omega_{*}=g N$. Darboux vector of the normal Fermi-Walker derivative is normal Fermi-Walker parallel in accordance with the normal Fermi- Walker derivative iff $g$ is constant.

Proof.

$$
\begin{array}{r}
\frac{\tilde{D} \omega^{*}}{\tilde{D} s}=\frac{d \omega^{*}}{d s}+f\left(\omega^{*} \wedge W\right), \\
\frac{d \omega^{*}}{d s}=\frac{d(g N)}{d s}=\frac{d(g)}{d s} N+\frac{d(N)}{d s} g, \\
\frac{d \omega^{*}}{d s}=\frac{d(g)}{d s} N+f g C .
\end{array}
$$

Also, since $f\left(\omega^{*} \wedge W\right)=-f g C, \quad \frac{\tilde{D} \omega^{*}}{\tilde{D} s}=\frac{d(g)}{d s} N$. Then, $\frac{\tilde{D} \omega^{*}}{\tilde{D} s}=0$ if and only if $g$ is constant.

Corollary 3.6. If $\beta(s)$ is constant precession curve, $\omega^{*}$ is normal Fermi-Walker parallel in accordance with the normal FermiWalker derivative. Infact, if $\beta(s)$ is constant precession curve, $f=$ constant, $\sigma=$ constant. Hence, $g$ is constant.

\section{Conclusion}

The Frenet frame is not non-rotating frame in accordance with Fermi-Walker derivative. Thus, we described a new Fermi-Walker derivative to be non-rotating frame of the Frenet frame and this new derivative called "normal Fermi-Walker derivative". Then, we showed that the Frenet frame is non-rotating frame in accordance with a new Fermi-Walker derivative. Otherwise, the adapted frame is not non-rotating frame in accordance with this new Fermi-Walker derivative. However, if a curve is general helix, the adapted frame is non-rotating frame in accordance with this new Fermi-Walker derivative.

Also, we defined normal Fermi-Walker parallelism, normal non-rotating frame, Darboux vector of normal Fermi-Walker derivative in accordance with the adapted frame in our study.

The Fermi-Walker derivative can be characterized by the first vector of other frames. In this way, the FermiWalker derivative can be redefined for different frames.

\section{Acknowledgment}

The first author would like to thank Tubitak-Bidep for their financial supports during her PhD studies.

\section{References}

[1] Balakrishnan R., Space curves, anholonomy and nonlinearity. Prama J. Phys. 64 (2005), no. 4, 607-615.

[2] Benn I.M. and Tucker R.W., Wave mechanics and inertial guidance. Phys. Rev. D. 39 (1989), no. 6, 1594-1601. 
[3] Berry M.V., Proc. Roy. Soc. London A. (1984), 392.

[4] Calin C. and Crasmareanu M., Slant Curves and Particles in three- dimensional Warped Products and their Lancret invariants. Bulletin of the Australian Mathematical Society. 88 (2013), no. 1, 128-142.

[5] Crasmareanu M. and Frigioiu C., Unitary vector fields are Fermi-Walker transported along Rytov-Legendre curves. Int. Journal of Geometric Methods in Modern Physics. 12 (2015), 1550111.

[6] Dandolof R., Berry's phase and Fermi-Walker parallel transport. Phys. Lett. A. 139 (1989), no. (1,2), 19-20.

[7] Fermi E. Atti Accad. Naz., Lincei Cl. Sci. Fiz. Mat. Nat. 31 (1922), 184-306.

[8] Hawking S.W. and Ellis G.F.R., The Large Scale Structure of Spacetime. Cambridge University Press., 1973.

[9] Karakus F. and Yayli Y., On the Fermi-Walker derivative and non-rotating frame. Int. Journal of Geometric Methods in Modern Physics. 2012, no. (9,8), 1250066.

[10] Karakus F. and Yayli Y., The Fermi- Walker derivative in Lie groups. Int. Journal of Geometric Methods in Modern Physics. 10 (2013), no. 7, Article ID 1320011:10p.

[11] Karakus F. and Yayli Y., The Fermi derivative in the hypersurfaces. Int. Journal of Geometric Methods in Modern Physics. 12 (2015), no. 1, Article ID 1550002:12p.

[12] Scofield P.D., Curves of Constant Precession. The American Mathematical Monthly 102 (1995), no. 6, 531-537.

[13] Uzunoglu B., Gok I. and Yayli Y., A new approach on curves of constant precession. Applied Mathematics and Computation. 275 (2016), 317-323.

\section{Affiliations}

ÖZGÜR KESKIN*

ADDRESS: Ankara University, Faculty of Science, Dept. of Mathematics, 06100, Tandoğan, Ankara-Turkey. E-MAIL: ozgur.keskin@ankara.edu.tr

YUSUF YAYLI

AdDress: Ankara University, Faculty of Science, Dept. of Mathematics, 06100, Tandoğan, Ankara-Turkey.

E-MAIL: yayli@science.ankara.edu.tr 